\title{
Discovery and timing of the first 8 gr8 Cygnus survey pulsars
}

\author{
G. H. Janssen ${ }^{1}$, B. W. Stappers ${ }^{2,1,3}$, R. Braun ${ }^{4,3}$, W. van $\operatorname{Straten}^{5}$, R. T. Edwards ${ }^{4}$, \\ E. Rubio-Herrera ${ }^{1}$, J. van Leeuwen ${ }^{3,1,6}$, and P. Weltevrede ${ }^{4,1}$ \\ 1 Astronomical Institute "Anton Pannekoek", University of Amsterdam, Kruislaan 403, 1098 SJ Amsterdam, The Netherlands \\ e-mail: g.h.janssen@uva.nl \\ ${ }^{2}$ University of Manchester, Jodrell Bank Observatory, Macclesfield Cheshire, SK11 9DL, UK \\ e-mail: Ben.Stappers@manchester.ac.uk \\ 3 Stichting ASTRON, Postbus 2, 7990 AA Dwingeloo, The Netherlands \\ 4 Australia Telescope National Facility - CSIRO, PO Box 76, Epping, NSW 1710, Australia \\ ${ }^{5}$ Centre for Astrophysics and Supercomputing, Swinburne University of Technology, PO Box 218 Hawthorn, VIC 3122, Australia \\ 6 Department of Physics and Astronomy, University of British Columbia, 6224 Agricultural Road, Vancouver B.C. V6T 1Z1, Canada
}

Received 8 December 2008 / Accepted 4 February 2009

\begin{abstract}
Context. Since 2004 we have been carrying out a pulsar survey of the Cygnus region with the Westerbork Synthesis Radio Telescope (WSRT) at a frequency of $328 \mathrm{MHz}$. The survey pioneered a novel interferometric observing mode, termed 8 gr8 (eight-grate), whereby multiple simultaneous digital beams provide high sensitivity over a large field of view.

Aims. Since the Cygnus region is known to contain OB associations, it is likely that pulsars are formed here. Simulations have shown that this survey could detect 70 pulsars, which would increase our understanding of the radio pulsar population in this region. We also aim to expand the known population of intermittent and rotating radio transient (RRAT)-like pulsars.

Methods. In this paper we describe our methods of observation, processing and data analysis, and we present the first results. Our observing method exploits the way a regularly spaced, linear array of telescopes yields a corresponding regularly spaced series of so-called "grating" beams on the sky. By simultaneously forming a modest number (eight) of offset digital beams, we can utilize the entire field of view of each WSRT dish, but retain the coherently summed sensitivity of the entire array. For the processing we performed a large number of trial combinations of period and dispersion measure (DM) using a computer cluster.

Results. In the first processing cycle of the WSRT 8gr8 Cygnus Survey, we have discovered three radio pulsars, with spin periods of $1.657,1.099$ and $0.445 \mathrm{~s}$. These pulsars have been observed on a regular basis since their discovery, both in a special follow-up programme as well as in the regular timing programme. The timing solutions are presented in this paper. We also discuss this survey method in the context of the SKA and its pathfinders.

Conclusions. We have found three new pulsars using the WSRT. Reprocessing and further analysis of the data will reveal dimmer pulsars, and RRAT-like or intermittent pulsars.
\end{abstract}

Key words. stars: neutron - pulsars: general

\section{Introduction}

In the last decade several pulsar surveys have been conducted using various telescopes. Many of those surveys have been extremely succesful, and altogether they have more than doubled the number of radio pulsars known in our galaxy; they have also found pulsars in some other nearby galaxies (e.g. Manchester et al. 2001; Crawford et al. 2001; Cordes et al. 2006; Hessels et al. 2008). Ongoing surveys are continuously contributing to the understanding of the characteristics of the whole pulsar population such as birth rates, velocity distribution, luminosity function, beaming fraction and magnetic field evolution. Studying large numbers of sources also provides insight into pulsar formation and evolution, and tells us about the local density and pressure environments in the interstellar medium and the magneticfield structure and electron distribution of the Galaxy itself. Furthermore, unique objects may be discovered, opening up new possibilities for pulsar science; e.g. the double pulsar (Burgay et al. 2003; Lyne et al. 2004), or possible PSR-BH systems.

In 2004 we began a special survey using the WSRT in The Netherlands. We developed a new beamforming technique that makes optimal use of the array configuration and multiband IF (intermediate frequency) system of the telescope for sensitive, wide-field pulsar surveys. We combine the field-of-view advantage of the small single dishes with the high sensitivity and angular resolution of a tied-array beam. This allows for a quick and accurate determination of the positions of all new pulsars. This survey covered a region containing the Cygnus superbubble and many OB associations (e.g. Uyanıker et al. 2001). In such regions of high star formation rate, it is reasonable to expect the discovery of significant numbers of, in particular, young pulsars.

In this paper we describe the setup and characteristics of the 8gr8 survey, and present the first results: the discovery of three pulsars.

\section{Observations and data analysis}

\subsection{8 gr8 survey: general introduction}

Radio telescopes that are designed to be used as interferometers are, in general, not useful for large area pulsar surveys. This is because they typically consist of several small dishes that are later combined, for imaging purposes, using a correlator. In order to obtain the sensitivity of the full collecting area of a synthesis 
telescope for pulsar observations it is necessary to combine the dishes "in phase", which means that the geometric and instrumental phase terms are corrected before the telescope signals are combined. These corrections are typically made for only one direction on the sky resulting in a "tied-array" beam with a width inversely proportional to the largest separation of the dishes in the array. In a typical WSRT observation of a known pulsar, all 14 of the $25 \mathrm{~m}$ diameter dishes are coherently combined to give a collecting area equivalent to a single dish of $94 \mathrm{~m}$ diameter. However, the size of the resultant beam on the sky corresponds to a dish with a diameter of $2.7 \mathrm{~km}$, the largest separation in the array. A simple calculation shows that the ratio of the field-ofview of a single $94 \mathrm{~m}$ dish to that resulting from the $2.7 \mathrm{~km}$ baseline of the WSRT is $\sim 1000$. A tied-array pulsar survey with the WSRT would therefore take $~ 1000$ times longer than a similar survey using a single $94 \mathrm{~m}$ dish.

We have developed a new method that allows us to overcome this limitation by making use of the fact that the first 12 WSRT telescopes, RT0 through RTB, can be placed at equal separations of $144 \mathrm{~m}$. The linear (East-West) nature of the WSRT array means that when the telescopes are added in phase to form a tiedarray, the instantaneous result is an elliptically shaped fan beam with a width (minor axis) inversely proportional to the separation of the furthest dishes, and a length (major axis) equivalent to the size of the primary beam. The equally spaced dishes produce a grating response on the sky, with parallel fan beams spaced at intervals of $c /(B v)$ radians, where $B$ is the projected baseline between the dishes and $v$ is the observing frequency (see Fig. 2a). We will refer to the collective fan beams in such a grating response as a grating group from now on. While these fan beams provide an increased field-of-view we can still do better by utilising the fact that the WSRT has eight independent signal chains allowing us to form eight simultaneous grating groups on the sky (Fig. 2a). Each of these grating groups is tuned to the same sky frequency but has different delay- and phase-tracking centres, offset by $c /(B v) / 8$, allowing us to almost fully tile the entire primary beam. Although the eight instantaneous grating groups do not fully sample the primary beam (12 groups would be required for that), the rotation of the Earth during the $\sim 7000 \mathrm{~s}$ observations lead to smearing of the point spread function, such that the variations in total integrated sensitivity over the beam are small (see Fig. 2b). The signals from the eight beams are later combined (using the appropriate linear combinations) in software to form elliptical subbeams which have dimensions equal to the beam size that would result from an equivalent synthesis observation. These subbeams are spaced to have them overlap at the half-power point of both axes. They tesselate out the entire primary beam and have a typical sensitivity that can be compared to that of a $74 \mathrm{~m}$ dish.

It is important at this stage to note some consequences of this method. The size of the primary beam, $c /(v D)$ where $D$ is the diameter of the dish, remains constant as a function of hour angle. However the separation and width, $11 B v / c$, of the fan beams depends on the length of the projected baseline, and thus is hour angle dependent. This means that the number of fan beams from a particular grating group which fall inside the primary beam changes from a minimum of one, when the projected baseline is equal to the dish diameter, up to a maximum of five at the zenith.

During an observation, the central fan beams track locations close to the primary beam phase centre which means that they "rotate" over source locations within the primary beam. When observing at large hour angles, the large width of the fan beams, combined with our approximately two hour observation duration, means that the sources remain predominantly in just one

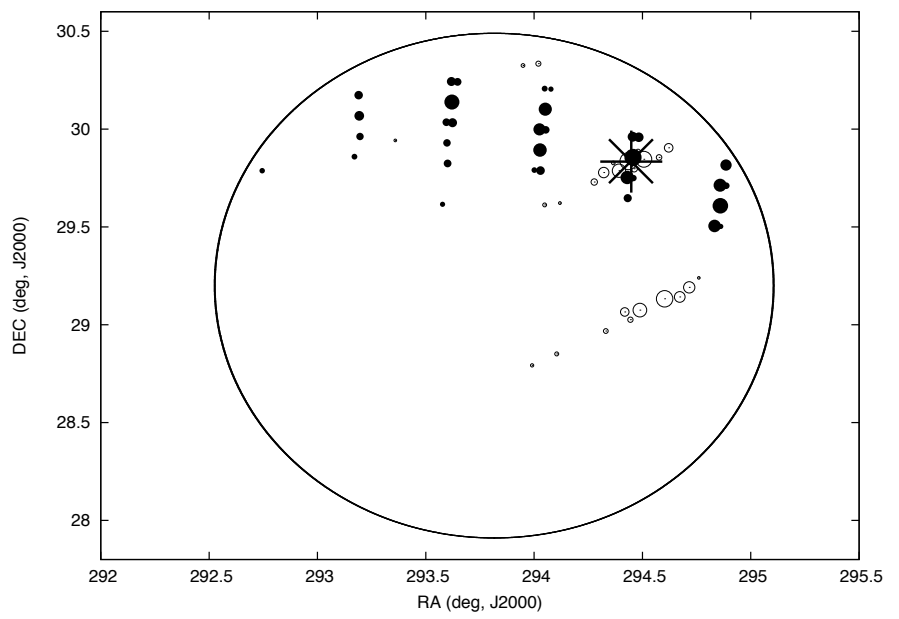

Fig. 1. Combined detection beamplot of the original and confirmation 8 gr8 observations in which PSR J1937+2950 was detected. The closed circles represent the subbeams in which the pulsar was detected in the first observation, and the open circles the detections in the confirmation observation. The sizes of the circles correspond to the $\mathrm{S} / \mathrm{N}$ of the detections. The grating effect as described in the text results in multiple detection-regions for each observation. Using the two observations at different hour angles, centred at the same position, allows for discriminating between the regions. The cross indicates the best-fit position of PSR J1937+2950 from the WSRT timing programme, see Table 1.

grating group. However at observations near the zenith, a source can pass through many grating groups. This is compensated in off-line processing, see Sect. 2.3. As each of the fan beams that make up a grating group has almost the same gain, any source which appears in one fan beam will also be present in the other fan beams from the same grating group inside the primary beam. An example of this is shown in Fig. 1. This means that one 8 gr8 observation, with observing parameters as described below, does not allow us to distinguish between the fan beams from the same grating group when determining the position of a new source.

This observing method has some useful advantages over single-telescope, single-receiver observations. While the width of the fan beams, or the number of fans from a grating group in the primary beam limit the accuracy to which the position of a new source can be determined, it is still better than using an equivalent observation with a single dish of the same size. A corollary of this is that any real source must be detected in more than one subbeam. In contrast, any source that appears with similar significance in a large number of subbeams is likely to be interference. This is because any near-field source should appear with equal sensitivity in all 8 of the bands. The almost two hour long observation time used for this survey means that, depending on hour angle, some positional discrimination is possible (see Fig. 1). To provide the ability for both direct confirmation of candidate pulsars and to improve positons for those candidates, we made a second observation of all pointings at different hour angles, leading to fan beams with different position angles within the primary beam. When combined with the first pointing this allows for further restriction of the likely position of the source. An example is shown in Fig. 1.

\subsection{Observations}

The region we have surveyed extends along the Galactic plane $40^{\circ}<l<100^{\circ},-0.25<b<7.25$, covering a total of 

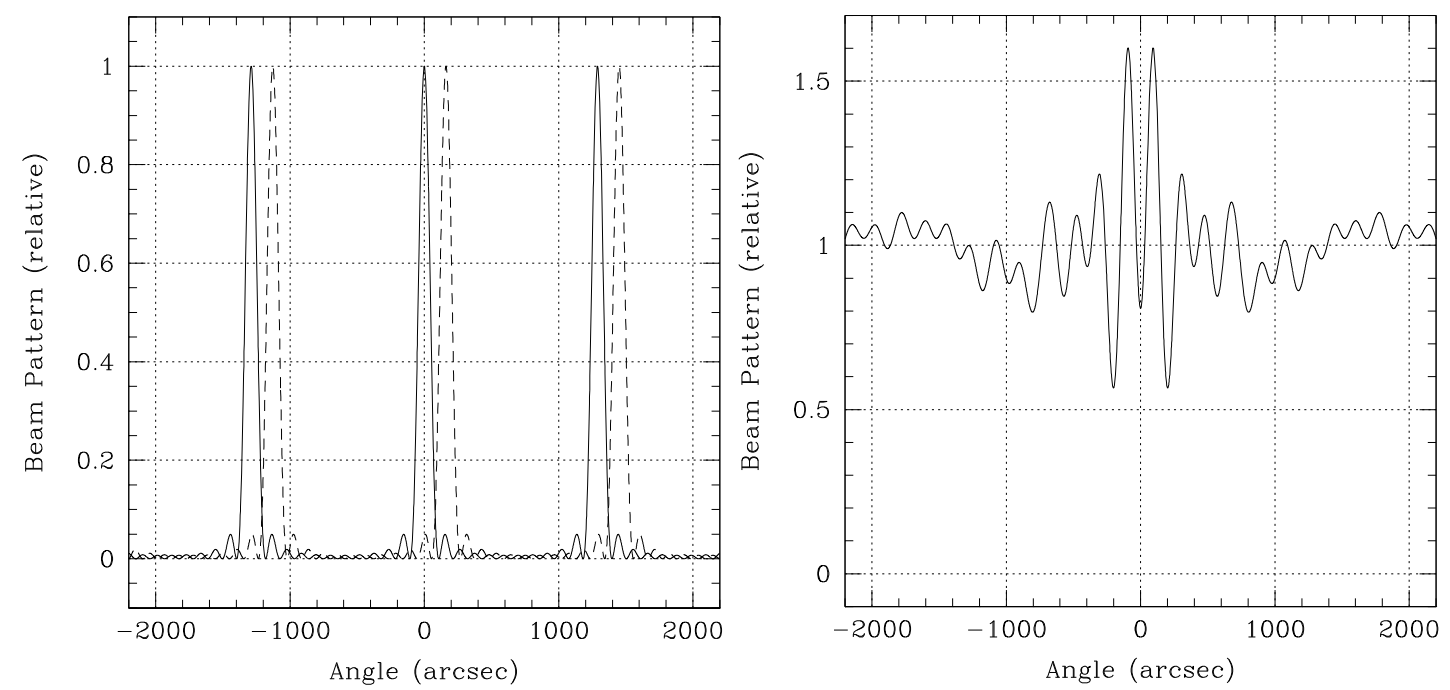

Fig. 2. a) Left: the instantaneous grating fan-beam response of the WSRT telescopes RT0 through RTB, added in phase, when all have a relative baseline of $144 \mathrm{~m}$. A cross-cut is displayed through the narrow dimension of the fan-beam. The overall taper corresponding to the telescope primary beam has not been included. One of the nearest neighbouring instantaneous grating groups is illustrated with the dashed line. A total of eight grating fan-beams provides complete coverage of the primary beam. Although the instantaneous fan-beams only overlap at about the $10 \%$ level, any finite integration time will broaden the effective beams, yielding a more nearly uniform combined sensitivity. b) Right: the relative sensitivity across the primary beam field-of-view after a $2 \mathrm{~h}$ integration with a total of eight grating fan-beams. The largest oscillations are within a few beamwidths of the overall field centre. These damp out at larger distances to a constant level.

450 square degrees. The survey was carried out at a central frequency of $328 \mathrm{MHz}$ with a bandwidth of $10 \mathrm{MHz}$. The field-ofview of the primary beam of the $25 \mathrm{~m}$ dishes at the WSRT at this frequency is 5.2 square degrees and, allowing for a small degree of overlap between the beams, we were able to span this region using 72 pointings spread over 12 days. We observed for $6872 \mathrm{~s}$ $\left(2^{23}\right.$ samples of $\left.819.2 \mu \mathrm{s}\right)$ and to be able to reach the high DMs that might be expected in the Cygnus region, we used 512 channels across the $10 \mathrm{MHz}$ bandwidth. Data rate limitations in the Pulsar Machine (PuMa) observing hardware (Voûte et al. 2002) meant that we had to reduce the sampling interval to a modest $819.2 \mu \mathrm{s}$, meaning that our sensitivity to the fastest rotating pulsars was reduced. However, as we were expecting, and aiming to detect, predominantly young pulsars in this region of the sky, this compromise was considered to be optimal. Motivated by the possibility that nearby pulsars might be missed in our survey due to scintillation, we observed each of the 72 pointings twice to increase the chance of detecting the source in a scintillation maximum. As mentioned before, this second data set also enabled instant confirmation of new pulsars.

The maximum sensitivity of the survey is shown in Fig. 3 using the survey parameters as described above, and represents an eight sigma detection. We consider three different Galactic latitudes, $b=0,3,6$ degrees, to indicate the increase in sensitivity as $T_{\text {sky }}$ decreases when we move away from the Galactic plane. We calculate the sensitivity for two representative DMs: 10 and $100 \mathrm{~cm}^{-3} \mathrm{pc}$. The calculation also includes the contribution to pulse broadening from scattering, as estimated using the DM-scatter broadening relation of Bhat et al. (2004). These curves represent the maximum sensitivity at the pointing centre and should be convolved with the curve shown in Fig. $2 \mathrm{~b}$ to give the sensitivity across the field-of-view.

Where possible, observations were scheduled at large hour angles so as to maximise the width of the fan beams and thus minimise the number of sub-beams that needed to be formed to tile out the primary beam. Despite this, the number of subbeams ranged from a minimum of 400 to a maximum of 2300 for the

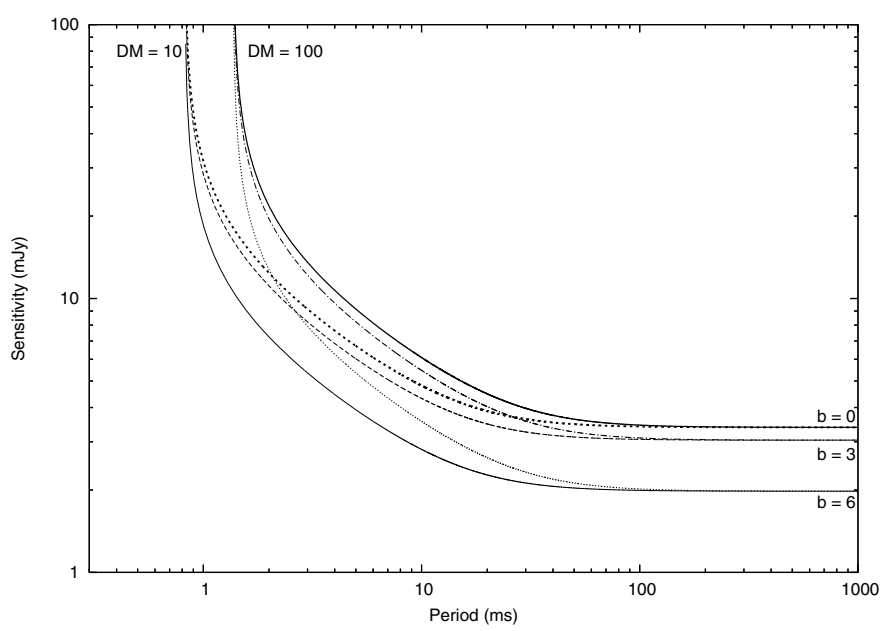

Fig. 3. Sensitivity curves, calculated using Eq. (A1.22) of Lorimer \& Kramer (2005), are shown for DMs of 10 and 100 and Galactic latitudes $b=0,3,6$ degrees, at the observing frequency of $328 \mathrm{MHz}$. The curves correspond to the minimum flux for detections of $8 \sigma$.

different pointings, resulting in a total of approximately 100000 subbeams across all 72 pointings. As each pointing was observed twice, and each subbeam has to be formed and then searched in the usual way individually, this results in a very large computational load.

The initial observations were completed in 2004 and the second set was finished in 2005. The massive computational load of searching more than 200000 subbeams meant that searching of the data could not begin until the arrival of the PuMa II cluster (Karuppusamy et al. 2008) in early 2005. The need for that cluster to be used for other observing programmes has meant that to date only a relatively low sensitivity pass through the data has been possible. In order to improve processing speed, the data were initially processed without any time domain interference 
removal nor correction for any baseline variations. The latter occurs when bright interference causes a readjustment in the level settings for packing the data into two bits. It results in steps in the data on timescales of tens of seconds which reduce our sensitivity to pulsars with periods longer than about a second. We have developed a new processing procedure which is able to correct for these steps and also is more robust to time domain interference. The significantly improved analysis, including single pulse searches, is currently ongoing and will be presented in a forthcoming paper.

\subsection{Survey processing}

Apart from the initial processing required to form the eight individual subbeams, the survey processing used the same software as was developed for the Parkes multibeam surveys (Manchester et al. 1996; Edwards et al. 2001). A modified form of the tree dedispersion algorithm (Taylor 1974) was applied to each of the data sets from the 8 beams. The total bandwidth of each beam was divided into smaller frequency subbands within which the dispersion could be assumed to be linear. For each of these subbands, a timeseries for all DMs up to the diagonal DM (i.e. the $\mathrm{DM}$ at which the dispersion delay across a frequency channel is about twice the sampling time) was formed. This resulted in a data set that can be thought of as a DM-frequency-time cube.

At this stage the eight fan beams were combined into subbeams by applying the appropriate geometric weighting over the total observing time before summing them, while still maintaining the DM-frequency-time cube structure. The appropriate weighting was determined by correcting for the fact that a particular position on the sky can move through different fan beams during an observation. The processing proceeded to cover the full range of the DM-frequency-time cube as follows; the dedispersion step was completed for 488 DMs separated by a DM step size of 0.696 up to the diagonal DM (of 340), resulting in a timeseries of $2^{23}$ samples for each DM. This time series was then Fourier transformed using a fast Fourier transform (FFT) routine. The resultant Fourier spectrum was cleaned by deleting frequencies known to correspond to interference and interpolated to recover spectral features lying between Fourier bins using the procedure described in Ransom et al. (2002). This process was repeated for spectra in which 2, 4, 8 and 16 harmonics were summed (Manchester et al. 1985) and for all DMs. All spectral features detected with a signal-to-noise ratio $(\mathrm{S} / \mathrm{N})$ of more than 7 were compared; those detected at multiple DMs and apparently related harmonics were grouped before selecting the combination of DM and spin frequency with the highest $\mathrm{S} / \mathrm{N}$ for each candidate.

For each of the resultant candidates, the partially dedispersed data were used as the start of a refinement procedure where the data were dedispersed and folded at DM and period values around those found in the spectral search. The range of period and DM searched were determined by assuming a maximum error of one Fourier bin. The partially dedispersed data were then divided into 8 to 32 subbands and 16 to 128 subintegrations, depending on the spin frequency and DM of the candidate, then folded and dedispersed at the period and DM values that gave the highest folded $\mathrm{S} / \mathrm{N}$, and stored for later inspection.

The lists of potential candidates in all subbeams of one pointing were collated, and those with high $\mathrm{S} / \mathrm{N}$ and detections in more than a single subbeam were stored. These detections were then compared to those made in the corresponding follow-up observation, and diagnostic plots, based on the aforementioned analysis of the matches were inspected. The best candidates were

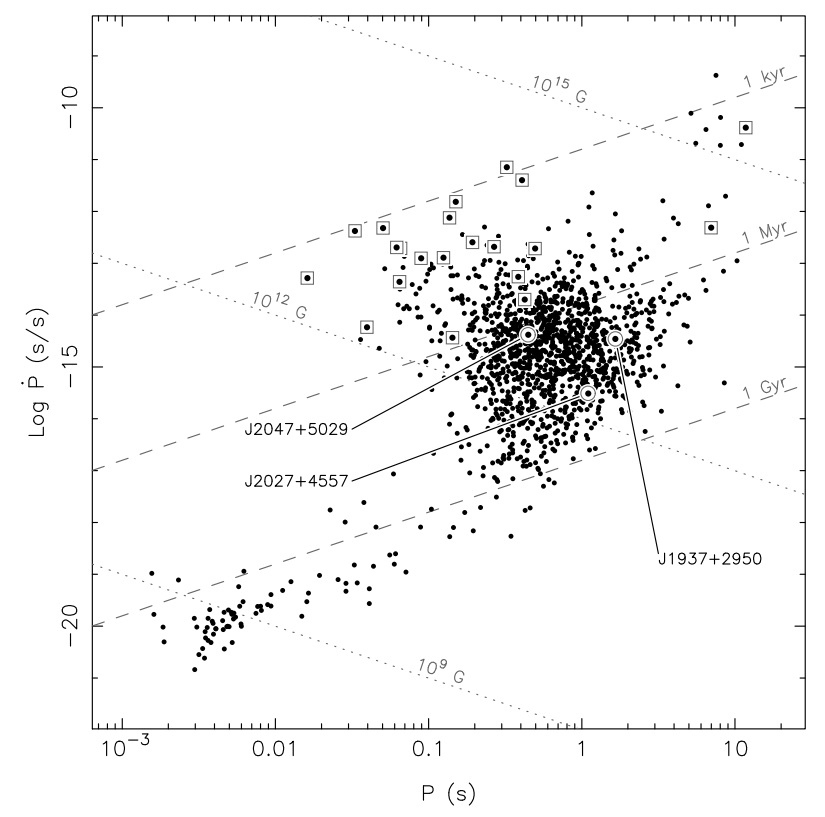

Fig. 4. Period-period derivative diagram for the three discovered pulsars. The pulsars labeled with a square are confirmed pulsar-SNR associations (ATNF pulsar catalogue ${ }^{3}$, Manchester et al. 2005).

reobserved in a follow-up timing programme, and three of those turned out to be pulsars.

\subsection{Follow-up timing}

The first follow-up series for the best pulsar candidates started in January 2006, and was again done in 8gr8 mode to be able to refine the positions. This also gave the advantage of covering a larger field-of-view, providing the possibility to use these observations as separate confirmation observations for any new candidate found in reprocessing of the original data. Sessions were separated by gaps, increasing in duration, to be able to phaseconnect the calculated times of arrival for each observation: in the first month of follow-up timing, observations were planned to take place in a sequence using day numbers $\{1,2,5,9,17,26\}$. Then for half a year, monthly observations in 8 gr8 mode were done, during which the positions were determined with a precision accurate enough to be included in the normal WSRT timing programme.

\subsection{Monitoring sources}

When the position for each of the three sources was believed to be known with high enough accuracy, they were included in regular timing observations at the WSRT, using the normal tied-array mode to optimise sensitivity. Furthermore, observations were done at additional frequencies to determine the DM more accurately, and to determine the spectral indices of the pulsars. As part of the normal timing programme, the new pulsars were observed approximately monthly using PuMa (Voûte et al. 2002). For observations at $328 / 374 \mathrm{MHz}$ we used two $10 \mathrm{MHz}$ bands; the observations centred at $1380 \mathrm{MHz}$ or $2270 \mathrm{MHz}$ used $80 \mathrm{MHz}$ of bandwidth, spread in 8 steps of $10 \mathrm{MHz}$ over a range of $160 \mathrm{MHz}$. The data were dedispersed and folded off-line, then integrated over frequency and time over the whole observation duration to obtain a single profile for each observation. Each profile was cross-correlated with a standard profile obtained via the summation of high signal-to-noise $(\mathrm{S} / \mathrm{N})$ profiles with the 


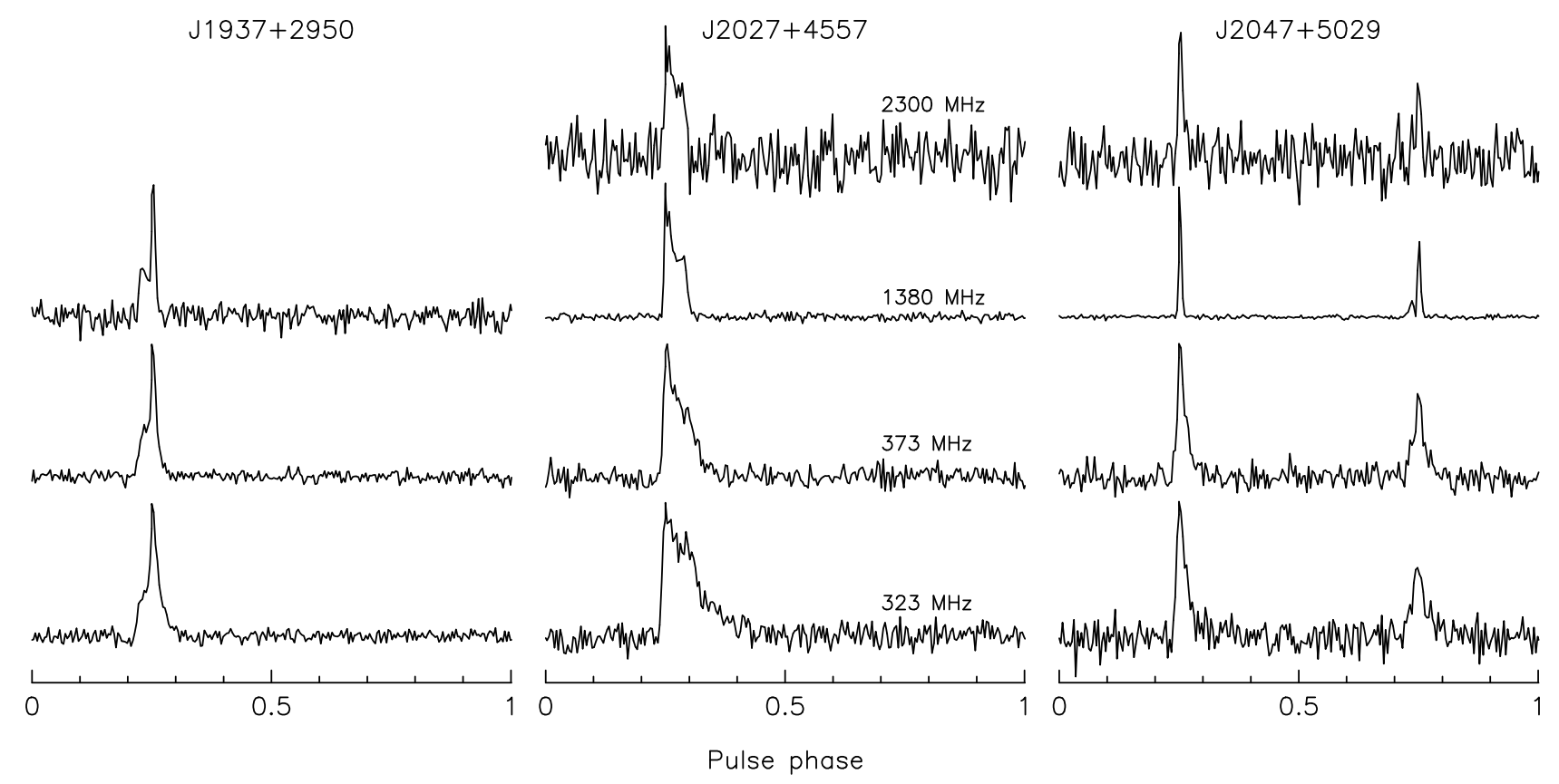

Fig. 5. WSRT standard profiles for PSR J1937+2950 (left), PSR J2027+4557 (middle) and PSR J2047+5029 (right). These templates have been generated using $\sim 15 \mathrm{~h}$ of timing data, except for the profiles of PSRs J2027+4557 and J2047+5029 at $2300 \mathrm{MHz}$, where $\sim 2 \mathrm{~h}$ of timing data was used.

corresponding observing frequency (Fig. 5), to calculate a time of arrival (TOA) for each observation. These were referred to local time generated by a H-maser clock at WSRT. The TOAs were converted to UTC using global positioning system (GPS) maser offset values measured at the observatory, and GPS to UTC corrections from the Bureau International des Poids et Mesures $(\mathrm{BIPM})^{1}$. Finally, the TOAs were converted to the Solar system barycentre using the JPL ephemeris DE4052. We used the new timing software package TEMPO2 (Hobbs et al. 2006) to analyse the data.

\section{Results}

The first three 8 gr8 pulsars were discovered as relatively bright sources in the original 8 gr8 observations. For each source, three observations were available at two or three different hour angles. As explained in Sect. 2, this allowed for removing any ambiguity in the position of the source, and measuring it accurately enough to observe the source in normal tied-array mode. To be able to obtain a coherent timing solution, a sequence of follow-up observations were done, as described in Sect. 2.5.

\subsection{PSR J1937+2950}

PSR J1937+2950 is a $1.657 \mathrm{~s}$ pulsar with a characteristic age of about $8 \mathrm{Myr}$. It was detected with high $\mathrm{S} / \mathrm{N}$ in the original pointing and two confirmation observations. It was included in the first session of follow-up observations, and being detected in every observation, easily included in normal timing mode observations. The spectral index (SI) was measured to be -3.3(2), calculated using the procedure described in Lorimer et al. (1995). The pulsar was not detected at $2.3 \mathrm{GHz}$, which is not surprising given the large SI. Two observations at $140 \mathrm{MHz}$ failed to detect the

\footnotetext{
1 http://www.bipm.org

2 ftp://ssd.jpl.nasa.gov/pub/eph/export/DE405/ de405iom.ps
}

pulsar despite its very large spectral index. Interstellar scattering effects for this pulsar are expected to be negligible (Cordes \& Lazio 2002). The non-detection at $140 \mathrm{MHz}$ may therefore indicate that the spectrum has flattened or turned over in this range. Summed profiles for the observed frequencies are shown in Fig. 5, and the timing solution is presented in Table 1.

\subsection{PSR J2027+4557}

PSR J2027+4557 has a period of 1.099 s. It is relatively old (57 Myr). It was independently detected in the GBT350 survey at Green Bank (Hessels et al. 2008). The timing solution is presented in Table 1 . The pulse profile exhibits quite a bit of scattering at lower frequencies, see Fig. 5, which is consistent with that expected for the line of sight towards this pulsar (Cordes \& Lazio 2002). The SI of this pulsar was measured to be -1.43(9), based on measurements of the mean flux density from observations at 328, 374, 1380, and $2300 \mathrm{MHz}$.

Of the three pulsars we discovered so far, PSR J2027+4557 is the brightest. Its individual radio pulses are easily detected, allowing for an analysis of subpulse modulation. The shape and intensity of the individual pulses is variable from pulse to pulse, and this can be visualised by making a pulse-stack: a plot in which successive pulses are plotted on top of each other. The modulation patterns in such plots can be extremely irregular, or very regular, depending on the pulsar. Repeating patterns of diagonal bands of emission, the so-called drifting subpulses (Drake \& Craft 1968), are observed for many pulsars in varying degrees of clarity (Weltevrede et al. 2006; Weltevrede et al. 2007). Subpulse modulation can be analysed by calculating the longitude resolved fluctuation spectrum (LRFS, Backer 1970) and the two-dimensional fluctuation spectrum (2DFS, Edwards \& Stappers 2002). For a detailed description of the methods used below, we refer to Weltevrede et al. (2006).

${ }_{3 \text { http://www.atnf.csiro.au/research/pulsar/psrcat/ }}$ 
Table 1. Timing solutions for the three pulsars.

\begin{tabular}{|c|c|c|c|}
\hline Pulsar name ......................... & $\mathrm{J} 1937+2950$ & $\mathrm{~J} 2027+4557$ & $\mathrm{~J} 2047+5029$ \\
\hline \multicolumn{4}{|l|}{ Fit and data-set } \\
\hline MJD range. & $53463-54751$ & $53462-54751$ & $53740-54751$ \\
\hline Number of TOAs $\ldots \ldots \ldots \ldots \ldots \ldots \ldots$ & 85 & 89 & 101 \\
\hline Rms timing residual $(\mathrm{ms}) \ldots \ldots \ldots \ldots \ldots$ & 2.8 & 0.6 & 1.3 \\
\hline Weighted fit........................ & $Y$ & $Y$ & $Y$ \\
\hline Reduced $\chi^{2}$ value $\ldots \ldots \ldots \ldots \ldots \ldots$ & 1.2 & 1.7 & 5.5 \\
\hline \multicolumn{4}{|l|}{ Measured Quantities } \\
\hline Right ascension, $\alpha(\mathrm{J} 2000) \ldots \ldots \ldots \ldots$ & $19^{\mathrm{h}} 37^{\mathrm{m}} 47^{\mathrm{s}} .603(14)$ & $20^{\mathrm{h}} 27^{\mathrm{m}} 16^{\mathrm{s}} .233(3)$ & $20^{\mathrm{h}} 47^{\mathrm{m}} 54.6400(4)$ \\
\hline Declination, $\delta(\mathrm{J} 2000) \ldots \ldots \ldots \ldots \ldots \ldots$ & $+29^{\circ} 50^{\prime} 01^{\mathrm{s}} 8(2)$ & $+45^{\circ} 57^{\prime} 57^{\prime \prime} .08(4)$ & $+50^{\circ} 29^{\prime} 38^{\prime \prime} 17(4)$ \\
\hline Pulse frequency, $v\left(\mathrm{~s}^{-1}\right) \ldots \ldots \ldots \ldots \ldots \ldots$ & $0.603344173844(9)$ & $0.909379126021(5)$ & $2.242431224778(16)$ \\
\hline First derivative of pulse frequency, $\dot{v}\left(\mathrm{~s}^{-2}\right) \ldots$ & $-1.2672(6) \times 10^{-15}$ & $-2.559(3) \times 10^{-16}$ & $-2.09976(8) \times 10^{-14}$ \\
\hline Second derivative of pulse frequency, $\ddot{v}\left(\mathrm{~s}^{-3}\right)$ & & & $-7.41(8) \times 10^{-24}$ \\
\hline Dispersion measure, DM $\left(\mathrm{cm}^{-3} \mathrm{pc}\right) \ldots \ldots$ & $113.80(2)$ & $229.594(11)$ & $107.676(5)$ \\
\hline \multicolumn{4}{|l|}{ Set Quantities } \\
\hline Epoch of frequency determination (MJD) .. & \multicolumn{2}{|c|}{54250.0} & \\
\hline Epoch of position determination (MJD) .... & \multicolumn{2}{|c|}{54250.0} & \\
\hline Epoch of DM determination (MJD)........ & \multicolumn{2}{|c|}{54250.0} & \\
\hline \multicolumn{4}{|l|}{ Derived Quantities } \\
\hline Characteristic age, $\tau$ (Myr) & 7.5 & 56.3 & 1.7 \\
\hline Surface magnetic field strength, $B\left(10^{12} \mathrm{G}\right)$ & 2.4 & 0.6 & 1.4 \\
\hline Distance, $d(\mathrm{kpc}) \ldots \ldots \ldots \ldots \ldots \ldots$ & $5.1(7)$ & $10.5(7.4-50.0)$ & $4.4(5)$ \\
\hline Energy loss, $\dot{E}_{\mathrm{rot}}\left(\mathrm{erg} \mathrm{s}^{-1}\right) \ldots \ldots \ldots \ldots \ldots$ & $3.0 \times 10^{31}$ & $9.2 \times 10^{30}$ & $1.9 \times 10^{33}$ \\
\hline Energy loss $/ d^{2}, \dot{E}_{\mathrm{rot}} / d^{2}\left(\mathrm{erg} \mathrm{s}^{-1} \mathrm{kpc}^{-2}\right) \ldots \ldots$ & $1.2 \times 10^{30}$ & $8.3 \times 10^{28}$ & $9.6 \times 10^{31}$ \\
\hline Mean flux density at $328 \mathrm{MHz},(\mathrm{mJy}) \ldots$. & $8.7(6)$ & $9.1(9)$ & $2.5(3)$ \\
\hline Mean flux density at $1380 \mathrm{MHz},(\mathrm{mJy}) \ldots$ & $0.08(2)$ & $1.34(13)$ & $0.38(4)$ \\
\hline Mean flux density at $2300 \mathrm{MHz},(\mathrm{mJy}) \ldots$ & & $0.32(9)$ & $0.22(13)$ \\
\hline Spectral index .................... & $-3.3(2)$ & $-1.43(9)$ & $-1.39(9)$ \\
\hline \multicolumn{4}{|l|}{ Assumptions } \\
\hline Clock correction procedure........... & \multicolumn{2}{|c|}{ TT(TAI) } & \\
\hline Solar system ephemeris model ........... & \multicolumn{2}{|c|}{ DE405 } & \\
\hline Model version number ... & \multicolumn{2}{|c|}{5.00} & \\
\hline
\end{tabular}

Note. Figures in parentheses are the nominal $1 \sigma$ TEMPO2 uncertainties in the least-significant digits quoted. The DM distances are estimated from the Cordes \& Lazio (2002) model.

Visual inspection of the individual pulses of PSR J2027+4557 reveal that pulses at the leading edge become stronger and weaker on a timescale of slightly more than 10 pulse periods, a clear sign that this pulsar could have drifting subpulses. The result of our fluctuation analysis of this pulsar can be seen in Fig. 6. The top panel shows the pulse profile, and the longitude-resolved modulation index is superimposed. The profile shows at least three components (at $158^{\circ}, 161^{\circ}$ and $172^{\circ}$ pulse longitude). The LRFS (middle panel) shows, for each pulse longitude, the Fourier transform of the intensities of the successive pulses, allowing us to determine whether the modulation is random or periodic. The most prominent feature is the patch of power associated with the leading component with a quasi-periodic frequency of about 0.07 cycles per period, thereby confirming the aforementioned intensity modulation with a period $P_{3}=14 \pm 1$ pulse periods. The lower panel shows the 2DFS of the leading component, which is sensitive to the slope of the drifting subpulses. The centroid of the power in between the two dashed lines is offset from the vertical axis, indicating that the subpulses drift towards later times in the pulse-stack. Other parts of the profile do not show evidence for drifting subpulses.

\subsection{PSR J2047+5029}

PSR J2047+5029 was first detected at its second harmonic. Separated by approximately 180 degrees, the pulsar has an interpulse that is about $50 \%$ as strong as the main pulse. Like PSR J2027+4557, PSR J2047+5029 is relatively bright at $21 \mathrm{~cm}$, having a SI of -1.39(9). It shows quite a bit of timing noise, see Fig. 7. There is a weak hint of periodicity in the timing noise, however it is too early to determine if there is any physical intrinsic cause for the timing noise, like a long-period binary orbit or small glitches.

\section{Association with supernova remnant HB21?}

PSR J2047+5029 is located along the same line of sight as the supernova remnant (SNR) HB 21 (Hanbury Brown \& Hazard 1953 , G89.0+4.7), with its position $\sim 0.5$ degrees away from the centre of the SNR as given by Green's catalogue 4 , see Fig. 8. HB 21 is irregularly shaped, probably due to a molecular cloud on one side that may have stopped the remnant from expanding symmetrically (Tatematsu et al. 1990).

HB 21 was searched for a radio pulsar before (Biggs \& Lyne 1996), but down to a limit of $13 \mathrm{mJy}$ (at $610 \mathrm{MHz}$ ) nothing was found. The search covered an area 0.5 degrees around the

${ }^{4}$ http://www.mrao.cam.ac.uk/surveys/snrs/ 

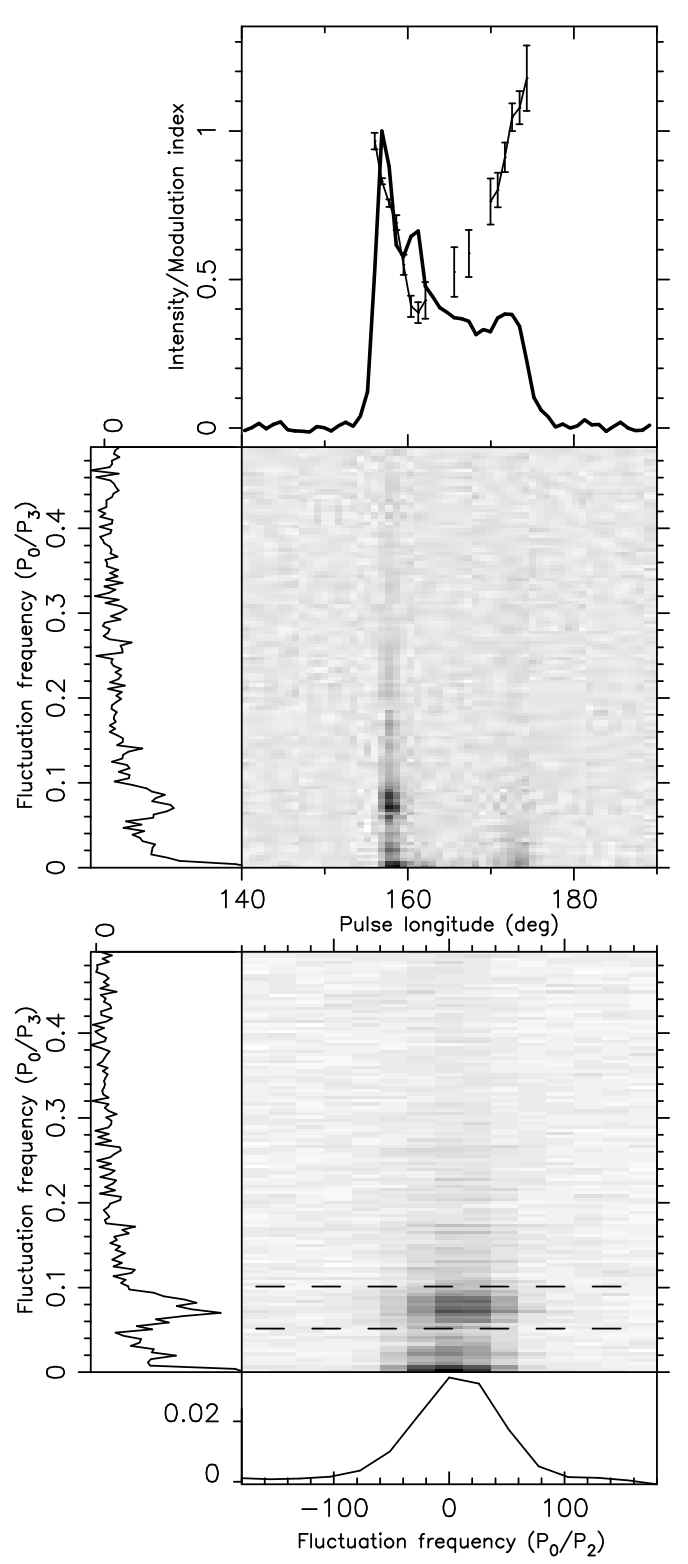

Fig. 6. Top panel: pulse profile and Longitude resolved modulation index for PSR J2027+4557. Middle panel: longitude resolved fluctuation spectrum (LRFS). Bottom panel: two-dimensional fluctuation spectrum (2DFS). These plots show one $50 \mathrm{~min}$. observation made using the new pulsar machine, PuMaII (Karuppusamy et al. 2008), which provided twice the bandwidth at $1380 \mathrm{MHz}$ and thereby increased the sensitivity to single pulses.

SNR centre, which probably just included the pulsar position. However, interpolating from our SI calculations, the expected flux density at $610 \mathrm{MHz}$ is $1.23 \mathrm{mJy}$, explaining why the pulsar was not detected in their survey. Lorimer et al. (1998) searched the whole remnant, also at $610 \mathrm{MHz}$, to a sensitivity limit of $0.66 \mathrm{mJy}$ (this number is calculated accounting for the duty cycle of the pulsar of $\sim 0.02$ ). Somewhat surprisingly, they did not detect PSR J2047+5029.

In general, the probability of having a pulsar along the line of sight towards a SNR by coincidence appears to be high, considering the number of pairs that are shown to be not associated (e.g. Gaensler \& Johnston 1995). Recent targeted searches have revealed a handful of pulsars in SNRs, but only a minority of

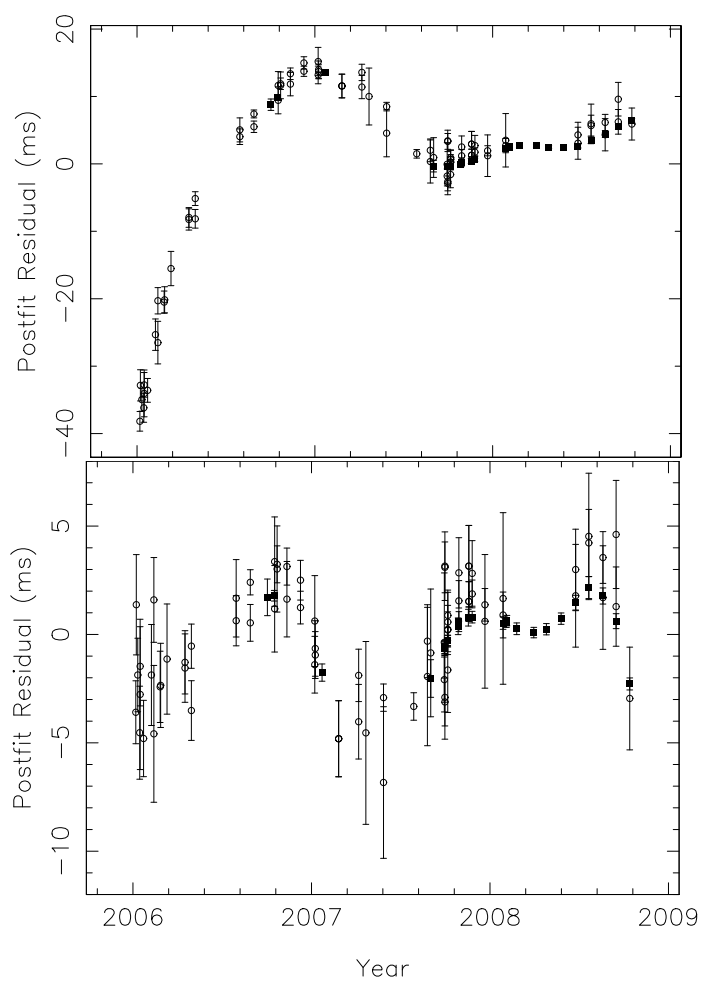

Fig. 7. Timing residuals for PSR J2047+5029. Top: solution including astrometric parameters, spin period and first spin period derivative. Bottom: solution also includes second derivative. Different symbols represent different observing frequencies; circles: 323 and $374 \mathrm{MHz}$, squares: $1380 \mathrm{MHz}$. For the parameters of this solution, see Table 1.

those are considered to be real associations (e.g. Camilo et al. 2002; Lorimer et al. 2006). Kaspi (1996) has proposed a number of questions to assess whether a pulsar is associated with a SNR, which we will address one by one below for this PSR-SNR combination. From the following, we conclude it is very unlikely that PSR J2047+5029 is associated with SNR HB 21:

Distance: Estimates of the distance of SNR HB21 vary a lot. References in Leahy (1987) give a range of 1.0 to $1.6 \mathrm{kpc}$, all derived using the surface brightness-diameter $(\Sigma-D)$ relation for SNRs. From radio continuum, HI and CO observations, Tatematsu et al. (1990) state that the SNR is part of the Cyg OB7 association, which has a known distance of 800(70) pc (Huang \& Thaddeus 1986). However, due to the lack of direct evidence of interaction of the SNR with the OB association, combined with a low X-ray surface brightness, the distance to HB 21 was proposed to be larger than $1.6 \mathrm{kpc}$ (references in Byun et al. 2006), and they combined all available distance estimates to arrive at a value of $1.7(5) \mathrm{kpc}$.

From the measurements of the DM of PSR J2047+5029, using the Cordes \& Lazio (2002) model, we derive a distance of 4.4(5) kpc. Although this may be overestimated due to unmodelled features in the DM model, the distance estimates for pulsar and remnant are at least different by a factor 3 . However, in Fig. 6 of Cordes \& Lazio (2002), a schematic overview is given of location of spiral arms in the Galaxy, which suggests that the pulsar, if at at distance of $4.4 \mathrm{kpc}$, is located in (or behind) the Perseus arm. This argues in favour of a coincident association. 


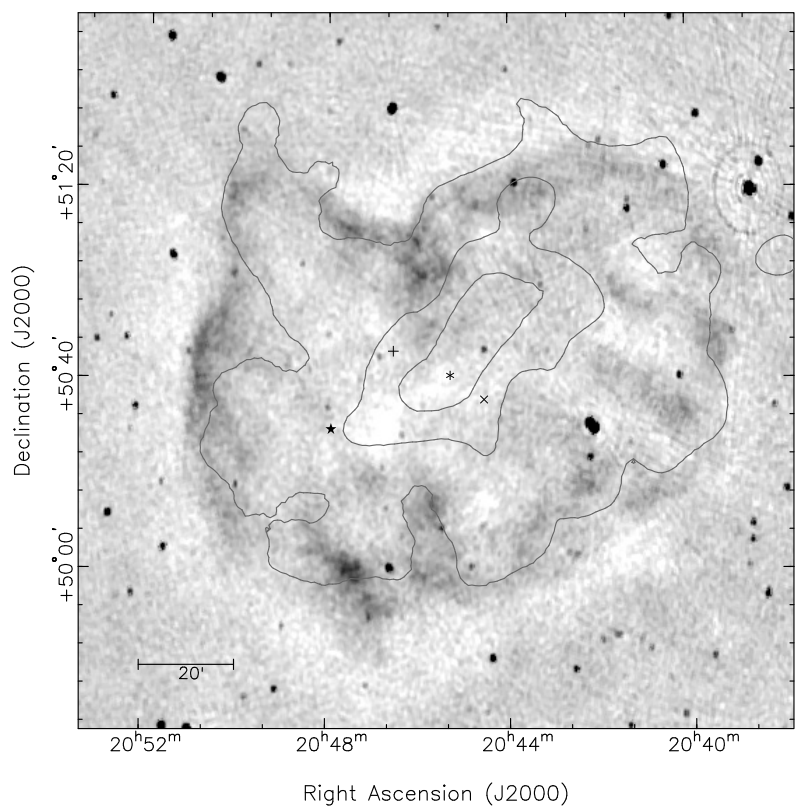

Fig. 8. Image of SNR HB 21, using WENSS radio images (Rengelink et al. 1997), and ROSAT contours (Leahy \& Aschenbach 1996). The symbols represent various centre positions of the SNR as quoted in the literature. (+ Green 2006; * Leahy \& Aschenbach 1996; and $\times$ Leahy 1987). The $\star$ represents the best position of PSR J2047+5029 from timing, see Table 1. The error on pulsar position is smaller than the size of the symbol.

Age: The age of the SNR was estimated by Leahy (1987) at 8000-15000 yr, by comparing the properties of the SNR (shock temperature, interior density) with models for SNR expansion into a 3-component medium. Leahy \& Aschenbach (1996) quote an age of 19000 years, based on a model for a SNR in a cloudy interstellar medium.

The characteristic age of the pulsar is two orders of magnitude larger, about $1.7 \mathrm{Myr}$. This apparent age conflict is yet another argument against association of the pulsar and the remnant. The characteristic age of a pulsar must be regarded as effectively only an upper limit to its real age, when a dipole braking model is assumed. However, requiring the pulsar age to be consistent with that of the SNR age and assuming the pulsar period derivative has not changed during its lifetime, leads to the conclusion that the birth period of the pulsar would have been similar to the current period to the extent of $10^{-3}$. As almost all pulsars that are confirmed to be associated with a SNR have spin periods less than about 140 ms (Lorimer et al. 1998; Kramer et al. 2003), the measured spin period for PSR J2047+5029 of $0.445 \mathrm{~s}$ is very unlikely to be close to its spin period at birth. So, though the characteristic age may not be used as a realistic age estimate, the pulsar must be quite old to have slowed down to this period, and we conclude that PSR J2047+5029 is too old to be associated with the SNR.

Velocity/Proper Motion: Most pulsars receive a kick velocity when born in a supernova event. The angular distance between the current position of a pulsar and the centre of its claimed corresponding SNR, combined with the estimated age and distance towards the combination, can therefore be used to calculate a minimum velocity needed for the pulsar to travel to its present position in its lifetime (e.g. Migliazzo et al. 2002).

For PSR J2047+5029, depending on the estimated age and distance of the remnant, the required velocity lies between 300 and $2000 \mathrm{~km} \mathrm{~s}^{-1}$, which is large but not unreasonable. So far we have not measured a proper motion signal in the timing residuals. Given the timing precision that we can achieve for this pulsar, and as we have been timing the pulsar for only a limited timespan, this is not surprising because the expected effect that a transverse velocity of $300-2000 \mathrm{~km} \mathrm{~s}^{-1}$ would have on the timing residuals is not measurable. It is therefore at this stage not possible to use proper motion as an argument for or against association between HB 21 and PSR J2047+5029.

Interaction with the remnant: There is no indication of emission at the pulsar position that might be from the pulsar itself, for a pulsar wind nebula, or from interaction with the SNR itself (see Fig. 8). However that may be expected from the flux limits of the WENSS survey (Rengelink et al. 1997). Unfortunately there is no radio image available with sensitivity to have detected the pulsar. Therefore this criterium is not useful for determining whether the pulsar and remnant are associated. We note also that there is no indication of either a pulsar or an associated wind nebula in the X-ray emission.

\section{Pulsar surveys with interferometers}

As previously discussed in Sect. 2, interferometers have not generally been used for pulsar surveys due to their limited survey speed, $\Sigma_{\mathrm{PSR}}$, which can be expressed as the product of instantaneous signal-to-noise squared with the field-of-view, or,

$\Sigma_{\mathrm{PSR}}=\left(\frac{A_{\mathrm{eff}}}{T_{\text {sys }}}\right)^{2} v^{2 \alpha} B W \Omega_{d^{2}}$,

where $A_{\text {eff }}$ is the effective collecting area in $\mathrm{m}^{2}, T_{\text {sys }}$ the system temperature, $v$ the observing frequency in $\mathrm{GHz}, \alpha$ the typical pulsar spectral index of about $-1.8, B W$ the observing bandwidth in $\mathrm{GHz}$ and $\Omega_{d^{2}}$ the field-of-view in square degrees. In view of the steep spectral indices of many pulsars, there is a natural speed advantage for moderately low frequency surveys, but there are often other considerations which dictate the optimum choice. In any case, this metric can be used to compare a number of existing and potential future pulsar surveys as shown in Table 2, where we note that higher values represent higher survey speeds.

The first two entries in the table document the very successful pulsar surveys carried out with the Parkes telescope at 70 and $20 \mathrm{~cm}$ (Manchester et al. 1996, 2001). A similar high survey speed was achieved in both bands by employing both multiple beams and much larger bandwidths at $20 \mathrm{~cm}$ relative to $70 \mathrm{~cm}$. The current Arecibo $20 \mathrm{~cm}$ multi-beam ALFA system (Cordes et al. 2006) offers a substantial improvement in survey speed over these previous efforts. In contrast, the current WSRT 8gr8 survey already achieves about twice the ALFA pulsar survey speed while employing only $10 \mathrm{MHz}$ of bandwidth (while $20 \mathrm{MHz}$ can now be accomodated with the PumaII upgrade in place).

The final three lines in the table offer guidelines for what will be necessary for future interferometric systems if they are to provide competitive pulsar survey capabilities. The ASKAP (Australian Square Kilometre Array Pathfinder) telescope is now under construction on the short-listed SKA site in Western Australia (Johnston et al. 2007). It will consists of 36 telescopes of $12 \mathrm{~m}$ diameter, fed in the $0.7-2 \mathrm{GHz}$ band by a focal plane array feed that will provide good performance over a 30 square degrees field-of-view. The ASKAP configuration is somewhat centrally concentrated but is distributed over a region of about $6 \mathrm{~km}$ 
Table 2. Pulsar survey speed comparison.

\begin{tabular}{lccccc}
\hline \hline Pulsar Survey & $\begin{array}{c}A_{\text {eff }} / T_{\text {sys }} \\
\left(\mathrm{m}^{2} / \mathrm{K}\right)\end{array}$ & $\begin{array}{c}v \\
(\mathrm{GHz})\end{array}$ & $\begin{array}{c}B W \\
(\mathrm{GHz})\end{array}$ & $\begin{array}{c}\Omega_{d^{2}} \\
\left(\mathrm{deg}^{2}\right)\end{array}$ & $\Sigma_{\mathrm{PSR}}$ \\
\hline Parkes 20 cm Multi-beam & 70 & 1.4 & 0.30 & 0.41 & 180 \\
Parkes 70 cm ........... & 32 & 0.41 & 0.03 & 0.39 & 290 \\
Arecibo 20 cm ALFA $\ldots$ & 805 & 1.4 & 0.3 & 0.02 & 1100 \\
WSRT 90 cm 8gr8 ...... & 31 & 0.33 & 0.01 & 4.2 & 2230 \\
ASKAP 35 cm 8k-beam. & 32 & 0.85 & 0.3 & 6 & 3300 \\
meerKAT 30 cm 1k-beam & 180 & 1.0 & 0.5 & 0.5 & 8300 \\
SKA 35 cm 1k-beam ..... & 2000 & 0.85 & 0.3 & 0.75 & $1.6 \times 10^{6}$ \\
\hline
\end{tabular}

in extent. In the table we have attributed only $50 \%$ of the total ASKAP sensitivity to a synthsized beam of about $100^{\prime \prime}$ diameter at $850 \mathrm{MHz}$. Since the ASKAP configuration is a randomised two dimensional one, rather than a pure "grating array" such as the WSRT, individual tied-array beams will need to be generated for each search direction in real time. Competitive pulsar surveys with ASKAP will require something like 8000 of these simultaneous tied-array beams to provide a significant improvement in survey speed. Together these would cover only about 6 of the 30 square degrees total field-of-view that is available. The meerKAT project plans to construct an SKA pathfinder in the other short-listed SKA site in Southern Africa ${ }^{5}$. It will consist of 80 telescopes of $12 \mathrm{~m}$ diameter, fed in the $0.75-2.3 \mathrm{GHz}$ band by a single pixel feed. The configuration is such that $70 \%$ of the collecting area will be contained in the inner $700 \mathrm{~m}$. As with ASKAP the non-grating array layout will require beam formation to be done in real time. The larger collecting area concentrated into a smaller region mean that meerKAT would require fewer beams, about 400, to be a competitive search instrument. For Table 2 we give numbers for a 1000 beam survey (J. Jones, priv. comm.). The much higher sensitivity expected for the SKA, of about $2000 \mathrm{~m}^{-2} / \mathrm{K}$ within the central few $\mathrm{km}$, permits very competitive pulsar surveys with many fewer simultaneous tiedarray beams. For example, 1000 beams would suffice to cover about 0.75 square degrees and yield about 3 orders of magnitude improvement in survey speed over the current state-of-the-art. We direct the reader to Smits et al. (2009) for a more complete discussion of SKA surveys.

\section{Conclusions}

Our new pulsar survey technique at the WSRT has resulted in the discovery of three new pulsars. In the first analysis of the data three new pulsars have been found. In contrast to the expectation of finding young pulsars, these are normal pulsars. However, the analysis procedure is being optimised and we expect finding many more pulsars and transients in the Cygnus region. The results and discussion of the full analysis of the survey will be presented in a forthcoming paper.

One of our newly discovered pulsars, PSR J1937+2950 has a SI of -3.3(2), showing that the survey is successful in finding steep-spectrum pulsars, and that there are potentially more to find. Somewhat surprisingly, two of the pulsars are brighter at $21 \mathrm{~cm}$, indicating that surveys of this region at that frequency may also still find quite bright pulsars. Moreover, the discovery of PSR J2027+4557 shows that it is still possible to find interesting single pulse sources with modern surveys. Following several evaluation criteria, we conclude that PSR J2047+5029, although having a position along the same line of sight as SNR HB 21, is not associated with this SNR.

Acknowledgements. The Westerbork Synthesis Radio Telescope is operated by ASTRON (Netherlands Foundation for Research in Astronomy) with support from the Netherlands Foundation for Scientific Research NWO. We thank the observers at WSRT for their help and hard work in planning and carrying out the vast number of observations that were necessary to complete this survey. We would like to thank Ger de Bruyn for making the WENSS images available, Ingrid Stairs for providing computing time at UBC, Sam Bates for providing Fig. 3 and Cees Bassa for combining the available archival WENSS and ROSAT images of SNR HB 21 into Fig. 8.

\section{References}

Backer, D. C. 1970, Nature, 227, 692

Bhat, N. D. R., Cordes, J. M., Camilo, F., Nice, D. J., \& Lorimer, D. R. 2004, ApJ, 605, 759

Biggs, J. D., \& Lyne, A. G. 1996, MNRAS, 282, 691

Burgay, M., D’Amico, N., Possenti, A., et al. 2003, Nature, 426, 531

Byun, D.-Y., Koo, B.-C., Tatematsu, K., \& Sunada, K. 2006, ApJ, 637, 283

Camilo, F., Manchester, R. N., Gaensler, B. M., \& Lorimer, D. R. 2002, ApJ, 579, L25

Cordes, J. M., \& Lazio, T. J. W. 2002, [arXiv: astro-ph/0207156]

Cordes, J. M., Freire, P. C. C., Lorimer, D. R., et al. 2006, ApJ, 637, 446

Crawford, F., Kaspi, V. M., Manchester, R. N., et al. 2001, ApJ, 553, 367

Drake, F. D., \& Craft, H. D. 1968, Nature, 220, 231

Edwards, R. T., \& Stappers, B. W. 2002, A\&A, 393, 733

Edwards, R. T., Bailes, M., van Straten, W., \& Britton, M. C. 2001, MNRAS, 326,358

Gaensler, B. M., \& Johnston, S. 1995, MNRAS, 277, 1243

Green, D. A. 2006, A Catalogue of Galactic Supernova Remnants, 2006 April Version (Cambridge: Mullard Radio Astronomy Observatory)

http: //www . mrao.cam.ac.uk/surveys/snrs/

Hanbury Brown, R., \& Hazard, C. 1953, MNRAS, 113, 123

Hessels, J. W. T., Ransom, S. M., Kaspi, V. M., et al. 2008, in AIP Conf. Series, ed. C. G. Bassa, Z. Wang, A. Cumming, \& V. M. Kaspi, 983, 613

Hobbs, G. B., Edwards, R. T., \& Manchester, R. N. 2006, MNRAS, 369, 655

Huang, Y. L., \& Thaddeus, P. 1986, ApJ, 309, 804

Johnston, S., Bailes, M., Bartel, N., et al. 2007, Proc. Astr. Soc. Aust., 24, 174

Karuppusamy, R., Stappers, B. W., \& Van Straten, W. 2008, PASP, 120, 191

Kaspi, V. M. 1996, in Pulsars: Problems and Progress, ed. S. Johnston, M. A.

Walker, \& M. Bailes (San Francisco: Astronomical Society of the Pacific), IAU Coll., 160, 375

Kramer, M., Lyne, A. G., Hobbs, G., et al. 2003, ApJ, 593, L31

Leahy, D. A. 1987, MNRAS, 228, 907

Leahy, D. A., \& Aschenbach, B. 1996, A\&A, 315, 260

Lorimer, D. R., \& Kramer, M. 2005, Handbook of Pulsar Astronomy (Cambridge University Press)

Lorimer, D. R., Yates, J. A., Lyne, A. G., \& Gould, D. M. 1995, MNRAS, 273, 411

Lorimer, D. R., Lyne, A. G., \& Camilo, F. 1998, A\&A, 331, 1002

Lorimer, D. R., Faulkner, A. J., Lyne, A. G., et al. 2006, MNRAS, 372, 777

Lyne, A. G., Burgay, M., Kramer, M., et al. 2004, Science, 303, 1153

Manchester, R. N., D’Amico, N., \& Tuohy, I. R. 1985, MNRAS, 212, 975

Manchester, R. N., Lyne, A. G., D’Amico, N., et al. 1996, MNRAS, 279, 1235

Manchester, R. N., Lyne, A. G., Camilo, F., et al. 2001, MNRAS, 328, 17

Manchester, R. N., Hobbs, G. B., Teoh, A., \& Hobbs, M. 2005, AJ, 129, 1993

Migliazzo, J. M., Gaensler, B. M., Backer, D. C., et al. 2002, ApJ, 567, L141

Ransom, S. M., Eikenberry, S. S., \& Middleditch, J. 2002, AJ, 124, 1788

Rengelink, R. B., Tang, Y., de Bruyn, A. G., et al. 1997, A\&AS, 124, 249

Smits, R., Kramer, M., Stappers, B. W., et al. 2009, A\&A, 493, 1161

Tatematsu, K., Fukui, Y., Landecker, T. L., \& Roger, R. S. 1990, A\&A, 237, 189

Taylor, J. H. 1974, A\&AS, 15, 367

Uyanıker, B., Fürst, E., Reich, W., Aschenbach, B., \& Wielebinski, R. 2001, A\&A, 371, 675

Voûte, J. L. L., Kouwenhoven, M. L. A., van Haren, P. C., et al. 2002, A\&A, 385, 733

Weltevrede, P., Edwards, R. T., \& Stappers, B. W. 2006, A\&A, 445, 243

Weltevrede, P., Stappers, B. W., \& Edwards, R. T. 2007, A\&A, 469, 607 